\title{
Correction to: Database and Expert Systems Applications
}

Sven Hartmann, Josef Küng, Gabriele Kotsis, A Min Tjoa, and Ismail Khalil

Correction to:

S. Hartmann et al. (Eds.): Database and Expert Systems Applications, LNCS 12392, https://doi.org/10.1007/978-3-030-59051-2

The paper "Bounded Pattern Matching Using Views" was accidentally published twice in the book "Database and Expert Systems Applications, Part II", and the paper "View Selection for Graph Pattern Matching" was omitted. This has been corrected. Each paper is now included once. 